\title{
How to teach fully illiterate adults to read
}

Régine Kolinsky, Cristina Carvalho, Isabel Leite, Ana Franco, \& José Morais

\begin{abstract}
About 750 million adults (15\%) worldwide lack any literacy skills, most because they lack adequate learning opportunities (UNESCO, 2016). In this chapter, we discuss how to teach to read to such people. We first examine scientific evidence suggesting that literacy acquisition does not radically differ as a function of age of acquisition. We then discuss the data relevant for designing effective methods aimed at teaching literacy to fully illiterate adults. We argue that the available adult data confirm those relative to teaching methods and learning processes that have been gathered on literacy acquisition by children. On the basis of those works we propose principles that should underlie any method aiming at rapidly developing basic literacy skills. Lastly, we present evidence (Kolinsky, Leite, Carvalho, Franco, \& Morais, submitted) suggesting that implementing these principles does indeed allow teaching illiterate adults to decode words and pseudo-words in a very short period of time.
\end{abstract}

Keywords: adult illiteracy, adult literacy, phonemic awareness, decoding, reading instruction. 